\title{
ADJOINTS OF SEMIGROUPS ACTING ON VECTOR-VALUED FUNCTION SPACES
}

\author{
BY \\ G. GREINER* \\ Universität Tübingen \\ Auf der Morgenstelle 10, D-7400 Tübingen, Germany \\ AND \\ J. M. A. M. VAN NEERVEN \\ Centre for Mathematics and Computer Science \\ P.O. Box 4079, 1009 AB Amsterdam, The Netherlands
}

\begin{abstract}
Let $T(t)$ be the translation group on $Y=C_{0}(\mathbb{R} \times K)=C_{0}(\mathbb{R}) \otimes C(K), K$ compact Hausdorff, defined by $T(t) f(x, y)=f(x+t, y)$. In this paper we give several representations of the sun-dial $Y \odot$ corresponding to this group. Motivated by the solution of this problem, viz. $Y^{\odot}=L^{1}(\mathbb{R}) \otimes M(K)$, we develop a duality theorem for semigroups of the form $T_{0}(t) \otimes$ id on tensor products $Z \otimes X$ of Banach spaces, where $T_{0}(t)$ is a semigroup on $Z$. Under appropriate compactness assumptions, depending on the kind of tensor product taken, we show that the sun-dial of $Z \otimes X$ is given by $Z^{\odot} \otimes X^{*}$. These results are applied to determine the sun-dials for semigroups induced on spaces of vector-valued functions, e.g. $C_{0}(\Omega ; X)$ and $L^{p}(\mu ; X)$.
\end{abstract}

\section{Introduction}

Suppose $\mu$ is a complex Borel measure of bounded variation on $\mathbb{R}$. For $t \in \mathbb{R}$ define the measure $\mu_{t}$ by $\mu_{t}(A)=\mu(A+t)$. Then a classical theorem due to Plessner [Pl] states that $\lim _{t \rightarrow 0}\left\|\mu-\mu_{t}\right\|=0$ if and only if $\mu \ll m$, where $m$

* This paper was written during a half-year stay at the Centre for Mathematics and Computer Science CWI in Amsterdam. I am grateful to the CWI and the Dutch National Science Foundation NWO for financial support.

Received August 16, 1990 
denotes the Lebesgue measure on $\mathbb{R}$. In Section 2 of this paper we derive the following analogue of this result for vector-valued measures: let $X$ be a Banach space and let $\mu$ be an $X$-valued Borel measure of bounded variation on $\mathbb{R}$, then $\lim _{t \rightarrow 0}\left\|\mu-\mu_{t}\right\|=0$ if and only if $\mu \in L^{1}(\mu ; X)$. By the Radon-Nikodym theorem, the case $X=\mathbb{C}$ reduces to Plessner's theorem.

In case $X=Y^{*}$ is a dual space, this result can be restated in terms of the translation group in the following way: if $T(t)$ denotes the translation group on $C_{0}(\mathbb{R} ; Y)$ then $L^{1}\left(\mathbb{R} ; Y^{*}\right)$ is the maximal space of strong continuity of the adjoint $T^{*}(t)$ of $T(t)$. Now both $C_{0}(\mathbb{R} ; Y)$ and $L^{1}\left(\mathbb{R} ; Y^{*}\right)$ can be written as certain tensor products, namely $C_{0}(\mathbb{R} ; Y)=C_{0}(\mathbb{R}) \tilde{\otimes}_{\varepsilon} Y$ and $L^{1}\left(\mathbb{R} ; Y^{*}\right)=L^{1}(\mathbb{R}) \tilde{\otimes}_{\pi} Y^{*}$ (the injective resp. projective tensor product), whereas the translation group on $C_{0}(\mathbb{R} ; Y)$ can be regarded as the tensor product $T_{0}(t) \otimes \mathrm{id}$, with $T_{0}(t)$ denoting translation on $C_{0}(\mathbb{R})$. This suggests the following question:

Given two Banach spaces $Z, X$, a strongly continuous semigroup $T_{0}(t)$ on $Z$, with $Z^{\odot}$ the maximal space of strong continuity of $T_{0}^{*}(t)$, when is it true that we have a formula like $(Z \otimes X)^{\odot}=Z^{\odot} \otimes X^{*}$ ?

Here $(Z \otimes X)^{\odot}$ is the maximal space of strong continuity of the adjoint of the induced semigroup $T_{0}(t) \otimes$ id on $Z \otimes X$. This question will be addressed in Section 3 for the injective and projective tensor product. These results can be applied to the vector-valued function spaces $L^{1}(\mu ; X)$ and $C_{0}(\Omega ; X)$. In order to treat also $L^{p}(\mu ; X)$ for $1<p<\infty$ we study in Section 4 the $l$-tensor product.

\section{Adjoint Semigroups}

In this section we will recall some of the standard results on adjoint semigroups. Proofs can be found in [BB, P]. Let $\left\{T_{0}(t)\right\}_{t \geq 0}$ (briefly, $T_{0}(t)$ ) be a $C_{0}$-semigroup on a Banach space $X$. The adjoint $T_{0}^{*}(t)$ of $T_{0}(t)$ is the semigroup on $X^{*}$ defined by $T_{0}^{*}(t):=T_{0}(t)^{*}$. From

$$
\left|\left\langle T_{0}^{*}(t) x^{*}-T_{0}^{*}(s) x^{*}, x\right\rangle\right| \leq\left\|x^{*}\right\|\left\|T_{0}(t) x-T_{0}(s) x\right\|
$$

one sees that the map $t \mapsto T_{0}^{*}(t) x^{*}$ is weak*-continuous for every $x^{*} \in X^{*}$. Hence if $X$ is reflexive, then $T_{0}^{*}(t)$ is weakly continuous and therefore strongly continuous. However in general $T_{0}^{*}(t)$ is not strongly continuous and it makes sense to define the sun-dual $X^{\odot}$ as the maximal subspace of $X^{*}$ on which $T_{0}^{*}(t)$ acts in a strongly continuous manner:

$$
X^{\odot}=\left\{x^{*} \in X^{*}: \lim _{t \downarrow 0}\left\|T_{0}^{*}(t) x^{*}-x^{*}\right\|=0\right\} .
$$


$X^{\odot}$ is a norm-closed, weak ${ }^{*}$-dense subspace of $X^{*}$. In fact, one has

$$
X^{\odot}=\overline{D\left(A_{0}^{*}\right)}
$$

where $A_{0}^{*}$ is the adjoint of the generator $A_{0}$ of $T_{0}(t)$; the closure is taken with respect to the norm-topology of $X^{*}$. Letting $R\left(\lambda, A_{0}\right)=\left(\lambda-A_{0}\right)^{-1}$ be the resolvent of $T_{0}(t)$, then $R\left(\lambda, A_{0}^{*}\right)=R\left(\lambda, A_{0}\right)^{*}$ and $D\left(A_{0}^{*}\right)=R\left(\lambda, A_{0}^{*}\right) X^{*}$. Clearly $X^{\odot}$ is invariant under $T_{0}^{*}(t)$. By restricting $T_{0}^{*}(t)$ to $X^{\odot}$ one obtains a strongly continuous semigroup on $X^{\odot}$, which we will denote $T_{0}^{\odot}(t)$. Let $A_{0}^{\odot}$ be its generator, then one can show that $A_{0}^{\odot}$ is precisely the part of $A_{0}^{*}$ in $X^{\odot}$.

Proposition 1.1: Let $k \geq 1$ and $\lambda \in \varrho\left(A_{0}\right)$. Then $X^{\odot}=\overline{R\left(\lambda, A_{0}^{*}\right)^{k} X^{*}}$.

In fact, $R\left(\lambda, A_{0}^{*}\right)^{k} X^{*}=D\left(\left(A_{0}^{*}\right)^{k}\right) \supset D\left(\left(A_{0}^{\odot}\right)^{k}\right)$ and the latter is norm-dense in $X^{\odot}$ since $A_{0}^{\odot}$ is a generator on $X^{\odot}$.

Starting from $T_{0}^{\odot}(t)$ one can repeat the duality construction and define $T_{0}^{\odot *}(t)$ and $X^{\odot \odot}=\left(X^{\odot}\right)^{\odot}$. The canonical map $j: X \rightarrow X^{\odot *}$,

$$
\left\langle j x, x^{\odot}\right\rangle:=\left\langle x^{\odot}, x\right\rangle
$$

is an embedding mapping $X$ into $X \odot \odot$. In case $j X=X^{\odot \odot}$ we say that $X$ is sun-reflexive with respect to $T_{0}(t)$. It is well-known that this is the case if and only if $R\left(\lambda, A_{0}\right)$ is weakly compact [Pa2].

The spectra of $A_{0}, A_{0}^{*}$ and $A_{0}^{\odot}$ coincide, see e.g. [Na, A-III]. This will be used throughout this paper, as well as more or less obvious identities like $R\left(\lambda, A_{0}\right)^{*} x^{\odot}$ $=R\left(\lambda, A_{0}^{\odot}\right) x^{\odot}\left(x^{\odot} \in X^{\odot}\right)$, etc.

\section{Translation in $C_{0}(\mathbb{R} ; X)$}

Let $X$ be a Banach space. On $C_{0}(\mathbb{R} ; X)$ the translation group $T(t)$ is defined by

$$
T(t) f(s)=f(t+s), \quad t \in \mathbb{R} .
$$

In this section we prove in two different ways that the sun-dual on $C_{0}(\mathbb{R} ; X)$ with respect to $T(t)$ is given by $L^{1}\left(\mathbb{R} ; X^{*}\right)$.

Let $M(\mathbb{R} ; X)$ denote the Banach space of all countably additive $X$-valued vector measures of bounded variation [DU]. If $X$ is the scalar field we simply write $M(\mathbb{R})$. For $\mu \in M(\mathbb{R} ; X)$ its variation $|\mu| \in M(\mathbb{R})$ is defined by

$$
|\mu|(E):=\sup _{\pi}\left\{\sum_{A \in \pi}\|\mu(E \cap A)\|\right\},
$$


where the supremum is taken over all partitions $\pi$ of $\mathbb{R}$ into finitely many disjoint subsets. If $\mu \in M(\mathbb{R} ; X)$ then $|\mu|$ is a finite positive measure in $M(\mathbb{R})$.

It is well-known (see [DU, pp. 181-182]) that the dual of $C_{0}(\mathbb{R} ; X)$ may be identified with $M\left(\mathbb{R} ; X^{*}\right)$ and we have

$$
\left\|\int_{\mathbb{R}} f d \mu\right\| \leq \int_{\mathbb{R}}\|f\| d|\mu|, \quad f \in C_{0}(\mathbb{R} ; X), \quad \mu \in M\left(\mathbb{R} ; X^{*}\right) .
$$

The space $L^{1}(\mathbb{R} ; X)$ can be identified with a closed subspace of $M(\mathbb{R} ; X)$ in the following way: for $h \in L^{1}(\mathbb{R} ; X)$ define $\mu_{h} \in M(\mathbb{R} ; X)$ by

$$
\mu_{h}(E):=\int_{E} h d \mu .
$$

Lemma 2.1: Suppose $\mu \in M(\mathbb{R} ; X)$ and $f \in C(\mathbb{R})$ with $\lim _{t \rightarrow-\infty} f(t)=0$. Define

$$
F(r):=\int_{-\infty}^{r} f(s) d \mu(s) .
$$

Then $F$ is strongly measurable.

Proof: In order to apply Pettis' measurability theorem [DS], we must show that (i) $F$ is weakly measurable, and (ii) $F$ is essentially separably-valued.

To prove (i) first let $m$ be a measure in $M(\mathbb{R})$. Then $\tilde{F}$ defined by

$$
\tilde{F}(r):=\int_{-\infty}^{r} f(s) d m(s)
$$

is measurable. (To see this, we may assume that $\mu$ and $f$ are real-valued, split $f=f_{+}-f_{-}$and $m=m_{+}-m_{-}$and note that if $f$ and $m$ are positive then $\tilde{F}$ is monotone, hence measurable). Using this we see that for any $x^{*} \in X^{*}$ the function

$$
r \mapsto\left\langle x^{*}, F(r)\right\rangle=\int_{-\infty}^{r} f(s) d\left\langle x^{*}, \mu\right\rangle(s)
$$

is measurable. This proves (i).

To prove (ii) define

$$
F_{1}(r):=\int_{-\infty}^{r}|f(s)| d|\mu|(s) .
$$

Since $F_{1}$ is monotone, $F_{1}$ is continuous except at a countable set $E$. For $r_{0} \notin E$, $r \in \mathbb{R}$ we have

$$
\left\|F(r)-F\left(r_{0}\right)\right\|=\left\|\int_{r_{0}}^{r} f(s) d \mu(s)\right\| \leq \int_{r_{0}}^{r}|f(s)| d|\mu|(s)=\left|F_{1}(r)-F_{1}\left(r_{0}\right)\right| .
$$

From this it follows that $F$ is continuous as well on $\mathbb{R} \backslash E$. Since moreover $\mathbb{R} \backslash E$ is separable it follows that $F(\mathbb{R} \backslash E)$ is separable. This proves (ii). 
THEOREM 2.2: If $T(t)$ is the translation group on $C_{0}(\mathbb{R} ; X)$ then $C_{0}(\mathbb{R} ; X)^{\odot}=$ $L^{1}\left(\mathbb{R} ; X^{*}\right)$.

Proof: First we prove that $L^{1}\left(\mathbb{R} ; X^{*}\right) \subset C_{0}(\mathbb{R} ; X)^{\odot}$. Let $x^{*} \in X^{*}$ and $f \in$ $L^{1}(\mathbb{R})$. Define $f \otimes x^{*} \in L^{1}\left(\mathbb{R} ; X^{*}\right)$ by

$$
\left(f \otimes x^{*}\right)(s)=f(s) x^{*} .
$$

Since translation is continuous on $L^{1}(\mathbb{R})$ it is clear that $f \otimes x^{*} \in C_{0}(\mathbb{R} ; X)^{\odot}$. Since the linear span of such functions is dense in $L^{1}\left(\mathbb{R} ; X^{*}\right)$, the inclusion $L^{1}\left(\mathbb{R} ; X^{*}\right) \subset C_{0}(\mathbb{R} ; X)^{\odot}$ follows. We now prove the reverse inclusion. Let $A$ be the generator of $T(t)$. Since $C_{0}(\mathbb{R} ; X)^{\odot}=\overline{D\left(A^{*}\right)}$ it suffices to prove the inclusion $R\left(\lambda, A^{*}\right) M\left(\mathbb{R} ; X^{*}\right) \subset L^{1}\left(\mathbb{R} ; X^{*}\right)$. For $f \in C_{0}(\mathbb{R} ; X), \mu \in M\left(\mathbb{R} ; X^{*}\right)$ we have

$$
\begin{aligned}
\left\langle R\left(\lambda, A^{*}\right) \mu, f\right\rangle=\langle\mu, R(\lambda, A) f\rangle & =\int_{\mathbb{R}} \int_{0}^{\infty} e^{-\lambda t} f(s+t) d t d \mu(s) \\
& =\int_{\mathbb{R}} \int_{s}^{\infty} e^{\lambda(s-t)} f(t) d t d \mu(s) \\
& =\int_{\mathbb{R}} \int_{-\infty}^{t} e^{\lambda(s-t)} f(t) d \mu(s) d t \\
& =\int_{\mathbb{R}} f(t) F(t) d t
\end{aligned}
$$

where

$$
F(t):=e^{-\lambda t} \int_{-\infty}^{t} e^{\lambda s} d \mu(s) .
$$

We will show that $F \in L^{1}\left(\mathbb{R} ; X^{*}\right)$. By Lemma $2.1, F$ is strongly measurable. But then we have

$$
\begin{aligned}
\left\|\int_{\mathbb{R}} F(t) d t\right\| & \leq \int_{\mathbb{R}}\|F(t)\| d t \\
& =\int_{\mathbb{R}} e^{-\lambda t}\left\|\int_{-\infty}^{t} e^{\lambda s} d \mu(s)\right\| d t \\
& \leq \int_{\mathbb{R}}\left[\int_{s}^{\infty} e^{\lambda(s-t)} d t\right] d|\mu|(s) \\
& =\frac{1}{\lambda}|\mu|(\mathbb{R})<\infty .
\end{aligned}
$$

This proves that $F \in L^{1}\left(\mathbb{R} ; X^{*}\right)$. But since we had

$$
\left\langle R\left(\lambda, A^{*}\right) \mu, f\right\rangle=\int_{\mathbb{R}} f(t) F(t) d t
$$


for all $f$ it is clear that $F=R\left(\lambda, A^{*}\right) \mu$ and the proof is finished.

For $\mu \in M(\mathbb{R} ; X)$ and $t \in \mathbb{R}$ we define $\mu_{t} \in M(\mathbb{R} ; X)$ by $\mu_{t}(E)=\mu(E+t)$, where $E \subset \mathbb{R}$ is measurable. According to Theorem 2.2 we have, in case $X$ is a dual space, that $\left\|\mu_{t}-\mu\right\| \rightarrow 0$ as $t \rightarrow 0$ if and only if $\mu \in L^{1}(\mathbb{R} ; X)$. This easily extends to the case where $X$ is an arbitrary Banach space.

Corollary 2.3: Let $\mu \in M(\mathbb{R} ; X)$. Then $\lim _{t \rightarrow 0}\left\|\mu_{t}-\mu\right\|=0$ if and only if $\mu \in L^{\mathbf{1}}(\mathbb{R} ; X)$.

Proof: Suppose $\left\|\mu_{t}-\mu\right\| \rightarrow 0$. Regarding $\mu$ as an $X^{* *}$-valued vector measure, it follows from Theorem 2.2 that $\mu \in L^{1}\left(\mathbb{R} ; X^{* *}\right)$. But since $\mu$ takes its values in $X$, the same must be true for the density function $h_{\mu}$ representing $\mu$. In fact, by the Lebesgue differentiation theorem [DU, III. 12.8] we have, for almost all $s$,

$$
h_{\mu}(s)=\lim _{\varepsilon \rightarrow 0} \frac{1}{\varepsilon} \int_{s}^{s+\varepsilon} h_{\mu}(\tau) d \tau=\lim _{\varepsilon \rightarrow 0} \frac{1}{\varepsilon} \mu(s, s+\varepsilon) .
$$

Since $\mu(s, s+\varepsilon) \in X$ for all $\varepsilon$ it follows that $h_{\mu}$ is $X$-valued. The converse assertion is clear.

In the scalar case it is well-known that $C_{0}(\mathbb{R})^{\odot \odot}=B U C(\mathbb{R})$, the Banach space of bounded, uniformly continuous functions on $\mathbb{R}$. As might be expected, in the vector-valued case we get $C_{0}(\mathbb{R} ; X)^{\odot \odot}=B U C\left(\mathbb{R} ; X^{* *}\right)$. This follows from Theorem 3.11 below.

We will now investigate the special case of Theorem 2.2 where $X=C(K)$ with $K$ compact Hausdorff (or $X=C_{0}(\Omega)$ with $\Omega$ locally compact Hausdorff). We have $C_{0}(\mathbb{R} ; C(K)) \simeq C_{0}(\mathbb{R} \times K)$. The following lemma is more or less standard. Lemma 2.4: Suppose $B \subset M(K)$ is separable. Then there is a positive $\mu \in$ $M(K)$ such that $\nu \ll \mu$ for all $\nu \in B$.

Proof: Let $\left(\nu_{n}\right)$ be a dense sequence in $B$ and define

$$
\mu:=\sum_{n=1}^{\infty} \frac{\left|\nu_{n}\right|}{2^{n}\left\|\nu_{n}\right\|} .
$$

Then $\nu_{n} \ll \mu$ for all $n$, so by closure also $\nu \ll \mu$ for all $\nu \in B$.

Identifying $C_{0}(\mathbb{R} ; C(K))$ with $C_{0}(\mathbb{R} \times K)$ the translation group from above is given by

$$
T(t) f(x, y)=f(x+t, y)
$$


The following result gives an alternative representation of the sun-dual of $C_{0}(\mathbb{R} \times$ $K$ ) with respect to this group. Lebesgue measure on $\mathbb{R}$ will be denoted by $m$; $\mu_{1} \otimes \mu_{2}$ denotes the product measure of two measures $\mu_{1}, \mu_{2}$.

THEOREM 2.5: $C_{0}(\mathbb{R} \times K)^{\odot}=\bigcup_{0 \leq \mu \in M(K)} L^{1}(\mathbb{R} \times K, m \otimes \mu)$.

Proof: By Theorem 2.2 we have $C_{0}(\mathbb{R} \times K)^{\odot}=L^{1}(\mathbb{R} ; M(K))$. But any $f \in$ $L^{1}(\mathbb{R} ; M(K))$ is essentially separably valued. Therefore without loss of generality we may assume that $\{f(t): t \in \mathbb{R}\}$ is a separable subset of $M(K)$. By Lemma 2.4 there is a positive $\mu \in M(K)$ such that $f(t) \ll \mu$ for all $f$. By the RadonNikodym theorem we may regard $f$ as an element of $L^{1}\left(\mathbb{R} ; L^{1}(K, \mu)\right)$. By the Fubini theorem, the latter is isometric to $L^{1}(\mathbb{R} \times K, m \otimes \mu)$. This proves the inclusion $C$. For the reverse inclusion, let $\mu \geq 0$ and pick $f \in L^{1}(\mathbb{R} \times K, m \otimes \mu)$. Approximate $f$ by a compactly supported $\tilde{f}$ in $C\left(\mathbb{R} \times K^{\prime}\right)$ and note that translation of $\tilde{f}$ is continuous in the $L^{1}$-norm.

By Theorem 2.5, any $\nu \in C_{0}(\mathbb{R} \times K)^{\odot}$ belongs to some $L^{1}(\mathbb{R} \times K, m \otimes \mu)$ with $\mu \geq 0$. We will now give an explicit description of a possible choice for $\mu$. For $\nu \in M(\mathbb{R} \times K)$ positive, define $\pi \nu \in M(K)$ by $\pi \nu(F):=\nu(\mathbb{R} \times F)$. Then for $f \in C(K)$ we have

$$
\int_{K} f(y) d \pi \nu(y)=\int_{K} \int_{\mathbb{R}} f(y) d \nu(x, y) .
$$

We need the following lemma.

Lemma 2.6: Let $\lambda, \mu$ and $\nu$ be positive measures in $M(\mathbb{R}), M(K)$ and $M(\mathbb{R} \times K)$ respectively. If $\nu \ll \lambda \otimes \mu$ then $\nu \ll \lambda \otimes \pi \nu$.

Proof: By assumption there is an $h \in L^{1}(\mathbb{R} \times K, \lambda \otimes \mu), h \geq 0$ a.e., such that $d \nu=h d(\lambda \otimes \mu)$. Define

$$
\begin{aligned}
& K_{0}:=\left\{y \in K: \int_{\mathbb{R}} h(x, y) d \lambda(x)=0\right\} \\
& K_{1}:=\left\{y \in K: \int_{\mathbb{R}} h(x, y) d \lambda(x)>0\right\} .
\end{aligned}
$$

By the Fubini theorem,

$$
\nu\left(\mathbb{R} \times K_{0}\right)=\int_{K_{0}} \int_{\mathbb{R}} h(x, y) d \lambda d \mu=0 .
$$


Now suppose $(\lambda \otimes \pi \nu)(A)=0$. We have to show that $\nu(A)=0$. But we have

$$
\begin{aligned}
0 & =(\lambda \otimes \pi \nu)(A)=\int_{K} \int_{\mathbb{R}} \chi_{A}(x, y) d \lambda(x) d(\pi \nu)(y) \\
& =\int_{K} \int_{\mathbb{R}} \int_{\mathbb{R}} \chi_{A}(x, y) h(z, y) d \lambda(x) d \lambda(z) d \mu(y) \\
& =\int_{K} \int_{\mathbb{R}} \chi_{A}(x, y)\left(\int_{\mathbb{R}} h(z, y) d \lambda(z)\right) d \lambda(x) d \mu(y) \\
& =\int_{K_{1}} \int_{\mathbb{R}} \chi_{A}(x, y)\left(\int_{\mathbb{R}} h(z, y) d \lambda(z)\right) d \lambda(x) d \mu(y) .
\end{aligned}
$$

Since $\int_{\mathbb{R}} h(z, y) d \lambda(z)>0$ for $y \in K_{1}$, we see that $A \cap\left(\mathbb{R} \times K_{1}\right)$ is a $\lambda \otimes \mu$ null set, hence also a $\nu$-null set (since by assumption $\nu \ll \lambda \otimes \mu$ ). Therefore $A \subset\left(A \cap\left(\mathbb{R} \times K_{1}\right)\right) \cup\left(\mathbb{R} \times K_{0}\right)$ is a $\nu$-null set.

Combination of Theorem 2.5 and Lemma 2.6 gives the following intrinsic characterization of those $\nu$ belonging to $C_{0}(\mathbb{R} \times K)^{\odot}$.

Theorem 2.7: $\nu \in C_{0}(\mathbb{R} \times K)^{\odot}$ if and only if $\nu \ll m \otimes \pi|\nu|$.

One might wonder whether there is a more direct proof of Theorem 2.7. Indeed such a proof can be given. What may be more surprising is that it is possible to re-deduce Theorem 2.2 as a corollary from 2.7. Since we think that this approach is interesting in its own right, we will carry it out.

Direct proof of Theorem 2.7: If $\nu \in L^{1}(\mathbb{R} \times K, m \otimes \pi|\nu|)$ then as in the proof of Theorem 2.5 we have $\nu \in C_{0}(\mathbb{R} \times K)^{\odot}$. The proof of the converse proceeds in two steps. For Borel measures $\mu$ on $\mathbb{R}$ and $\nu$ on $\mathbb{R} \times K$ define the 'convolution' $\mu * \nu$ on $\mathbb{R} \times K$ by

$$
\int_{\mathbb{R} \times K} f d(\mu * \nu)=\int_{\mathbb{R} \times K} \int_{\mathbb{R}} f(x+t, y) d \mu(t) d \nu(x, y) .
$$

Now let $\nu \in C_{0}(\mathbb{R} \times K)^{\odot}$.

STEP 1: For $T>0$ let $m_{[0, T]}$ be the Borel measure on $\mathbb{R}$ defined by $m_{[0, T]}(E)=$ $m(E \cap[0, T])$. For $f \in C_{0}(\mathbb{R} \times K)$ and $T>0$ we have

$$
\begin{aligned}
\left\langle\frac{1}{T} \int_{0}^{T} T^{*}(t) \nu d t, f\right\rangle & =\left\langle\nu, \frac{1}{T} \int_{0}^{T} T(t) f d t\right\rangle \\
& =\frac{1}{T} \int_{\mathbb{R} \times K} \int_{0}^{T} f(x+t, y) d t d \nu(x, y) \\
& =\frac{1}{T}\left\langle m_{[0, T]} * \nu, f\right\rangle
\end{aligned}
$$


This shows that the equality

$$
\frac{1}{T} \int_{0}^{T} T^{*}(t) \nu d t=\frac{1}{T} m_{[0, T]} * \nu
$$

holds. We claim that

$$
m_{[0, T]} * \nu \ll m *|\nu|
$$

Indeed, let $E$ be measurable such that $(m *|\nu|)(E)=0$. This means by definition that

$$
\int_{\mathbb{R} \times K} \int_{\mathbb{R}} \chi_{E}(x+t, y) d m(t) d|\nu|(x, y)=0 .
$$

It follows that

$$
\int_{\mathbb{R} \times K} \int_{0}^{T} \chi_{E}(x+t, y) d t d|\nu|(x, y)=0 .
$$

Hence

$$
\chi_{E}(x+t, y)=0, \quad m_{[0, T]} \otimes|\nu|-\text { a.e. }
$$

From this it is clear that also

$$
\chi_{E}(x+t, y)=0, \quad m_{[0, T]} \otimes \nu-\text { a.e. }
$$

Rewriting this in terms of convolution, this is the same as $\left(m_{[0, T]} * \nu\right)(E)=0$. Our claim is proved. By now we have shown that

$$
\frac{1}{T} \int_{0}^{T} T^{*}(t) \nu d t \ll m *|\nu|
$$

Since by assumption

$$
\lim _{T \downarrow 0} \frac{1}{T} \int_{0}^{T} T^{*}(t) \nu d t=\nu
$$

strongly and since obviously $\{\mu: \mu \ll m *|\nu|\}$ is closed, it follows that $\nu \ll m *|\nu|$.

STEP 2: We claim that $m *|\nu|=m \otimes \pi|\nu|$. Let $\pi: \mathbb{R} \times K \rightarrow K$ be projection onto the second coordinate. We claim that the following equality holds:

$$
\int_{\mathbb{R} \times K} f \circ \pi d|\nu|=\int_{K} f d \pi|\nu| .
$$

Indeed, by the Riesz Representation Theorem the linear functional on $C(K)$ defined by

$$
f \mapsto \int_{\mathbb{R} \times K} f \circ \pi d|\nu|
$$


is represented by some $\mu \in C(K)^{*}$ and it is straightforward to check that $\mu=\pi|\nu|$. This proves the claim.

For $A \subset \mathbb{R} \times K$ measurable, put

$$
A_{y_{1}}:=A \cap\left\{(x, y) \in \mathbb{R} \times K: y=y_{1}\right\}
$$

Using our claim and the translation invariance of the Lebesgue measure $m$ we see

$$
\begin{aligned}
(m *|\nu|)(A) & =\int_{\mathbb{R} \times K} \int_{\mathbb{R}} \chi_{A}(x+t, y) d m(t) d|\nu|(x, y) \\
& =\int_{\mathbb{R} \times K} m(A-x)_{y} d|\nu|(x, y) \\
& =\int_{\mathbb{R} \times K} m(A)_{y} d|\nu|(x, y) \\
& =\int_{K} m(A)_{y} d \pi|\nu|(y) \\
& =\int_{K} \int_{\mathbb{R}} \chi_{A}(t, y) d m(t) d \pi|\nu|(y) \\
& =\int_{\mathbb{R} \times K} \chi_{A}(t, y) d(m \otimes \pi|\nu|)(t, y) \\
& =(m \otimes \pi|\nu|)(A) .
\end{aligned}
$$

This shows that $m *|\nu|=m \otimes \pi|\nu|$. Combining this with Step 1 we see that $\nu \ll m \otimes \pi|\nu|$ as was to be proved.

Second proof of Theorem 2.2: Let $X$ be an arbitrary Banach space. By the Banach-Alaoglu theorem the dual unit ball $K:=B_{X^{*}}$ is weak ${ }^{*}$-compact. The map $i: X \rightarrow C(K)$ defined by $i x\left(x^{*}\right)=\left\langle x^{*}, x\right\rangle$ is an isometric embedding. Let $\tilde{i}: C_{0}(\mathbb{R} ; X) \rightarrow C_{0}(\mathbb{R} ; C(K))=C_{0}(\mathbb{R} \times K)$ be the induced embedding. In this way we may regard $C_{0}(\mathbb{R} ; X)$ as a closed, translation invariant subspace of $C_{0}(\mathbb{R} \times K)$. Let $y^{\odot} \in C_{0}(\mathbb{R} ; X)^{\odot}$. We must show: $y^{\odot} \in L^{1}\left(\mathbb{R} ; X^{*}\right)$. By the extension theorem for adjoint semigroups $[\mathrm{Ne}], y^{\odot}$ can be extended to an element $\nu$ of $C_{0}(\mathbb{R} \times K)^{\odot}$. By Theorem 2.7 there is a density function $g \in L^{1}(\mathbb{R} \times K, m \otimes$ $\pi|\nu|)=L^{1}\left(\mathbb{R} ; L^{1}(K, \pi|\nu|)\right)$ representing $\nu$. We claim that $y^{\odot}=(\tilde{i})^{*} \nu$ can be regarded as an element of $L^{1}\left(\mathbb{R} ; X^{*}\right)$. To see this, let $f \in C_{0}(\mathbb{R} ; X)$ be arbitrary 
and note that

$$
\begin{aligned}
\int_{\mathbb{R}} f(\tau) d y^{\odot}(\tau) & =\left\langle y^{\odot}, f\right\rangle=\langle\nu, \tilde{i}(f)\rangle \\
& =\int_{\mathbb{R}}(\tilde{i}(f))(\tau) d \nu(\tau)=\int_{\mathbb{R}} g(\tau)(\tilde{i}(f))(\tau) d \tau \\
& =\int_{\mathbb{R}} g(\tau) i(f(\tau)) d \tau=\int_{\mathbb{R}} i^{*}(g(\tau)) f(\tau) d \tau
\end{aligned}
$$

Hence $y^{\odot}$ can be represented by $\tilde{g}$, defined by $\tilde{g}(t):=i^{*}(g(t))$. Since $i^{*}(g(t)) \in X^{*}$ for all $t \in \mathbb{R}$ we see that $y^{\odot} \in L^{1}\left(\mathbb{R} ; X^{*}\right)$ and the claim is proved.

\section{The Injective and Projective Tensor Product}

Throughout this section $X$ and $Z$ will denote non-zero Banach spaces. We assume either both to be real or complex. $Z \otimes X$ denotes the algebraic tensor product (cf. [S1]).

The $\pi$-norm on $Z \otimes X$, often called the projective norm, is described most conveniently by its unit ball, which by definition is the convex closure of the set $B_{Z} \otimes B_{X}$, where $B_{Z}$ and $B_{X}$ are the unit balls of $Z$ and $X$ respectively. An analytic expression for the $\pi$-norm is given as follows:

$$
\|u\|_{\pi}=\inf \left\{\sum_{i=1}^{n}\left\|z_{i}\right\|,\left\|x_{i}\right\|: u=\sum_{i=1}^{n} z_{i} \otimes x_{i}\right\}, \quad u \in Z \otimes X
$$

The $\pi$-tensor product $Z \tilde{\otimes}_{\pi} X$ is the completion of $Z \otimes X$ with respect to this norm. Sometimes it is denoted by $Z \hat{\otimes} X$. The standard example for the $\pi$-tensor product is the following. Let $Z$ be a space $L^{1}(\mu)$, where $\mu$ is some positive measure and $X$ an arbitrary Banach space. Then $L^{1}(\mu) \tilde{\otimes}_{\pi} X$ can be identified in a canonical way with the space $L^{1}(\mu, X)$ of all $X$-valued Bochner integrable functions.

An element $u=\sum_{i=1}^{n} z_{i} \otimes x_{i} \in Z \otimes X$ can (algebraically) be identified with an operator $T_{u} \in \mathcal{L}\left(Z^{*}, X\right)$ by the formula

$$
T_{u} z^{*}=\sum_{i=1}^{n}\left\langle z^{*}, z_{i}\right\rangle x_{i}
$$

The $\varepsilon$ - or injective norm on $Z \otimes X$ is the norm induced by the operator norm 
on $\mathcal{L}\left(Z^{*}, X\right)$. Thus for $u=\sum_{i=1}^{n} z_{i} \otimes x_{i}$ the $\varepsilon$-norm is given by

$$
\begin{aligned}
\|u\|_{\varepsilon} & =\sup \left\{\left\|\sum_{i=1}^{n}\left\langle z^{*}, z_{i}\right\rangle x_{i}\right\|:\left\|z^{*}\right\| \leq 1\right\} \\
& =\sup \left\{\left|\sum_{i=1}^{n}\left\langle z^{*}, z_{i}\right\rangle\left\langle x^{*}, x_{i}\right\rangle\right|:\left\|z^{*}\right\| \leq 1,\left\|x^{*}\right\| \leq 1\right\} .
\end{aligned}
$$

The completion of $Z \otimes X$ with respect to this norm is denoted by $Z \tilde{\otimes}_{\varepsilon} X$. It is called the $\varepsilon$ - or injective tensor product of $Z$ and $Y$. Some authors denote it by $Z \check{\otimes} X$. The standard example is as follows: let $Z:=C_{0}(\Omega), \Omega$ locally compact and $X$ be an arbitrary Banach space. Then $C_{0}(\Omega) \tilde{\otimes}_{\varepsilon} X$ can be identified with $C_{0}(\Omega ; X)$.

It is well-known that dual spaces of tensor products can be identified with certain operator ideals. For $u^{*} \in\left(Z \tilde{\otimes}_{\varepsilon} X\right)^{*}$ or $u^{*} \in\left(Z \tilde{\otimes}_{\pi} X\right)^{*}$, define $T_{u^{*}} \in$ $\mathcal{L}\left(Z, X^{*}\right)$ by

$$
\left\langle u^{*}, u\right\rangle=\sum_{i=1}^{n}\left\langle T_{u^{*}} z_{i}, x_{i}\right\rangle,
$$

where $u=\sum_{i=1}^{n} z_{i} \otimes x_{i} \in Z \otimes X$. In particular, the dual of $Z \tilde{\otimes}_{\pi} X$ can be identified with the space $\mathcal{L}\left(Z, X^{*}\right)$. On the other hand, the dual of $Z \tilde{\otimes}_{\varepsilon} X$ can be identified with the set of all integral operators $Z \rightarrow X^{*}$ [DU], which we denote by $\mathcal{L}^{i}\left(Z, X^{*}\right)$.

A bounded linear operator $T \in \mathcal{L}(Z)$ induces a linear operator $T \otimes \mathrm{id}: Z \otimes X \rightarrow$ $Z \otimes X$ by the formula

$$
(T \otimes \mathrm{id})(z \otimes x):=T z \otimes x .
$$

The operator $T \otimes$ id is bounded for both the $\varepsilon$ - and the $\pi$-norm. In fact, in both cases one has $\|T \otimes \mathrm{id}\|=\|T\|$. The unique continuous extensions to $Z \tilde{\otimes}_{\varepsilon} X$ and $Z \tilde{\otimes}_{\pi} X$ will be denoted by $T \tilde{\otimes}_{\varepsilon}$ id and $T \tilde{\otimes}_{\pi}$ id respectively.

LEMMA 3.1: $\sigma\left(T \tilde{\otimes}_{\varepsilon}\right.$ id $)=\sigma\left(T \tilde{\otimes}_{\pi}\right.$ id $)=\sigma(T)$.

Proof: We prove a slightly more general result: Suppose $\|\cdot\|$ is a reasonable crossnorm (in the sense of [DU; Def. VIII.1.1]) on $Z \otimes X$ with the additional property that every bounded linear operator $T: Z \rightarrow Z$ extends to a bounded linear operator $T \tilde{\otimes}$ id on the completion $Z \tilde{\otimes} X$ of $Z \otimes X$ with respect to $\|\cdot\|$. Then $\sigma(T \tilde{\otimes} \mathrm{id})=\sigma(T)$.

$$
\sigma(T \tilde{\otimes} \text { id }) \subset \sigma(T): \text { Suppose } \lambda-T \text { is invertible. Then }(\lambda-T)^{-1} \tilde{\otimes} \text { id is a }
$$
bounded operator on $Z \tilde{\otimes} X$ and it is obvious that on the dense subspace $Z \otimes X$, 
$(\lambda-T)^{-1} \otimes \mathrm{id}$ is a two-sided inverse for $\lambda-(T \otimes \mathrm{id})$. By density it follows that $(\lambda-T)^{-1} \tilde{\otimes} \mathrm{id}=(\lambda-(T \tilde{\otimes} \mathrm{id}))^{-1}$, so $\lambda \in \varrho(T \tilde{\otimes} \mathrm{id})$.

$\sigma(T) \subset \sigma(T \tilde{\otimes} \mathrm{id}):$ Suppose $\lambda \in \sigma(T)$. If $\lambda \in \sigma_{a p}(T)$, the approximate point spectrum of $T$ (cf. [Na]), then by definition we can choose an approximate eigenvector $\left(z_{n}\right)_{n=1}^{\infty}$, i.e., $\left\|z_{n}\right\|=1$ for all $n$ and

$$
\lim _{n \rightarrow \infty}\left\|T z_{n}-\lambda z_{n}\right\|=0
$$

We claim that $\left(z_{n} \otimes x\right)_{n=1}^{\infty}$ is an approximate eigenvector of $T \tilde{\otimes}$ id for every norm-1 vector $x \in X$. Indeed, we have $\left\|z_{n} \otimes x\right\|=\left\|z_{n}\right\|\|x\|=1$ and moreover

$$
\begin{aligned}
\left\|(T \tilde{\otimes} \mathrm{id})\left(z_{n} \otimes x\right)-\lambda\left(z_{n} \otimes x\right)\right\| & =\left\|\left(T z_{n}-\lambda z_{n}\right) \otimes x\right\| \\
& =\left\|T z_{n}-\lambda z_{n}\right\|\|x\| \rightarrow 0, \quad n \rightarrow \infty .
\end{aligned}
$$

Thus $\lambda \in \sigma\left(T \tilde{\otimes}\right.$ id). If $\lambda \in \sigma(T) \backslash \sigma_{a p}(T)$ then the range of $\lambda-T$ cannot be dense. According to the Hahn-Banach theorem, $\lambda \in \sigma_{p}\left(T^{*}\right)$. Choose a norm-1 vector $z^{*}$ such that $T^{*} z^{*}=\lambda z^{*}$. We claim that $\lambda \in \sigma_{p}\left((T \tilde{\otimes} \mathrm{id})^{*}\right)$ with eigenvector $z^{*} \otimes x^{*}$, where $x^{*} \neq 0$ is arbitrary in $X^{*}$. Indeed, for any $z \otimes x$ we have

$$
\begin{aligned}
\left\langle(T \tilde{\otimes} \mathrm{id})^{*}\left(z^{*} \otimes x^{*}\right), z \otimes x\right\rangle & =\left\langle z^{*} \otimes x^{*}, T z \otimes x\right\rangle \\
& =\left\langle z^{*}, T z\right\rangle\left\langle x^{*}, x\right\rangle \\
& =\left\langle T^{*} z^{*}, z\right\rangle\left\langle x^{*}, x\right\rangle \\
& =\lambda\left\langle z^{*}, z\right\rangle\left\langle x^{*}, x\right\rangle \\
& =\lambda\left\langle z^{*} \otimes x^{*}, z \otimes x\right\rangle .
\end{aligned}
$$

The claim now follows from a density argument. Hence $\lambda \in \sigma\left((T \tilde{\otimes} \mathrm{id})^{*}\right)=$ $\sigma(T \tilde{\otimes} \mathrm{id})$. The second inclusion is proved and the lemma follows.

Given a strongly continuous semigroup $T_{0}(t)$ on $Z$ with generator $A_{0}$ then $T(t):=T_{0}(t) \otimes$ id extends to a one-parameter semigroup of bounded linear operators on $Z \tilde{\otimes}_{\epsilon} X$ and $Z \tilde{\otimes}_{\pi} X$ respectively. In fact it is easy to see that it is strongly continuous as well. Moreover, spectrum and resolvent can be described. We state these facts in the following proposition, in which $\tilde{\otimes}$ denotes either the $\varepsilon$ - or the $\pi$-tensor product. 
Proposition 3.2: $T(t)$ is a strongly continuous semigroup. If we denote its generator by $A$ then $\sigma(A)=\sigma\left(A_{0}\right)$. For $\lambda$ in the resolvent set we have $R(\lambda, A)=$ $R\left(\lambda, A_{0}\right) \tilde{\otimes}$ id.

Proof: By the spectral mapping formula (cf. [Na]) we have

$$
\sigma\left(R\left(\lambda, A_{0}\right)\right) \backslash\{0\}=\left(\lambda-\sigma\left(A_{0}\right)\right)^{-1}
$$

and similarly for $A$. Hence, to prove the first assertion, we see that it suffices to show that $\sigma(R(\lambda, A))=\sigma\left(R\left(\lambda, A_{0}\right) \tilde{\otimes}\right.$ id $)$, but this follows from the previous lemma. The second assertion is obvious (e.g. apply a density argument).

Our next aim is to give a description of the adjoints of $T(t)$ and $R(\lambda, A)$. In order to do this, we identify the dual spaces of $Z \tilde{\otimes}_{\pi} X$ and $Z \tilde{\otimes}_{\varepsilon} X$ with $\mathcal{L}\left(Z, X^{*}\right)$ and $\mathcal{L}^{i}\left(Z, X^{*}\right)$ respectively. Given a bounded operator on $Z$, we want to determine the adjoint of $S \tilde{\otimes}$ id, where $\tilde{\otimes}$ is either $\tilde{\otimes}_{\varepsilon}$ or $\tilde{\otimes}_{\pi}$. Given $z \otimes x \in Z \otimes X$ and $R \in \mathcal{L}\left(Z, X^{*}\right)$ or $R \in \mathcal{L}^{i}\left(Z, X^{*}\right)$, then

$$
\langle R,(S \tilde{\otimes} \mathrm{id})(z \otimes x)\rangle=\langle R,(S z) \otimes x\rangle=\langle R S z, x\rangle=\langle R S, z \otimes x\rangle .
$$

This shows that we have $(S \tilde{\otimes} \mathrm{id})^{*}(R)=R S$. We summarize this observation in the following proposition.

Proposition 3.3: The adjoint operators $T^{*}(t)$ and $R(\lambda, A)^{*}: \mathcal{L}\left(Z, X^{*}\right) \rightarrow$ $\mathcal{L}\left(Z, X^{*}\right)$ are given as follows :

$$
\begin{aligned}
& T^{*}(t)(S)=S T_{0}(t), \quad S \in \mathcal{L}\left(Z, X^{*}\right) ; \\
& R(\lambda, A)^{*}(S)=S R\left(\lambda, A_{0}\right), \quad S \in \mathcal{L}\left(Z, X^{*}\right) .
\end{aligned}
$$

The same assertions are valid for the $\tilde{\otimes}_{\varepsilon}$ tensor product, with $\mathcal{L}\left(Z, X^{*}\right)$ replaced by $\mathcal{L}^{i}\left(Z, X^{*}\right)$.

Let us recall that the integral operators form a two-sided operator ideal, i.e. given $R \in \mathcal{L}^{i}\left(Z, X^{*}\right)$ and bounded linear operators $S_{1} \in \mathcal{L}(Z)$ and $S_{2} \in \mathcal{L}\left(X^{*}\right)$ then $S_{2} \circ R \circ S_{1}$ is integral as well and $\left\|S_{2} \circ R \circ S_{1}\right\|_{i} \leq\left\|S_{2}\right\| \cdot\|R\|_{i} \cdot\left\|S_{1}\right\|$. Here $\|\cdot\|_{i}$ is the norm induced by $\left(Z \tilde{\otimes}_{\varepsilon} X\right)^{*}$.

Both dual spaces $\mathcal{L}\left(Z, X^{*}\right)$ and $\mathcal{L}^{i}\left(Z, X^{*}\right)$ contain $Z^{*} \otimes X^{*}$ as a subspace. In order to identify the closure of $Z^{*} \otimes X^{*}$ with appropriate subspaces of $\mathcal{L}\left(Z, X^{*}\right)$ and $\mathcal{L}^{i}\left(Z, X^{*}\right)$ respectively we make for the rest of Section 3 the following assumption: 
Assumptron 3.4: $Z^{*}$ has the approximation property (a.p.).

The classical Banach spaces $\ell^{p}, C_{0}(\Omega), L^{p}(\mu)$ satisfy Assumption 3.4. $Z^{*}$ having the a.p. implies that the closure of $Z^{*} \otimes X^{*}$ in $\mathcal{L}^{i}\left(Z, X^{*}\right)$ can be identified with $Z^{*} \tilde{\otimes}_{\pi} X^{*}$. Operators belonging to this closure are called nuclear operators. Moreover, since $Z^{*}$ has the a.p., so does $Z$ [DU]. The latter implies that the closure of $Z^{*} \otimes X^{*}$ in $\mathcal{L}\left(Z, X^{*}\right)$, which is $Z^{*} \tilde{\otimes}_{\varepsilon} X^{*}$, is precisely the set of all compact operators from $Z$ into $X^{*}$.

Now we are going to show that in case of sun-reflexivity the sun-dual of the $\varepsilon$-tensor product can be described easily. We already noted in section 1 that a semigroup is sun-reflexive if and only if the resolvent of the generator is weakly compact.

Theorem 3.5: Let $Z$ be sun-reflexive with respect to $T_{0}(t)$. Then the sun-dual of the semigroup $T(t)$ induced on $Z \tilde{\otimes}_{\varepsilon} X$ is the closure in $Z^{*} \tilde{\otimes}_{\pi} X^{*}$ of $Z^{\odot} \otimes X^{*}$. Proof: Given $z^{*} \in Z^{*}$ and $x^{*} \in X^{*}$ then $T^{*}(t)\left(z^{*} \otimes x^{*}\right)=\left(T_{0}^{*}(t) z^{*}\right) \otimes x^{*}$. It follows that

$$
\left\|T^{*}(t)\left(z^{*} \otimes x^{*}\right)-z^{*} \otimes x^{*}\right\|=\left\|\left(T_{0}^{*}(t) z^{*}-z^{*}\right)\right\| \cdot\left\|x^{*}\right\|
$$

This shows that if $z^{*} \in Z^{\odot}$ then $z^{*} \otimes x^{*} \in\left(Z \tilde{\otimes}_{\varepsilon} X\right)^{\odot}$. Hence also the closed linear subspace of $Z^{*} \tilde{\otimes}_{\pi} X^{*}$ generated by $\left\{z^{*} \otimes x^{*}: z^{*} \in Z^{\odot}, x^{*} \in X^{*}\right\}$ is contained in $\left(X \tilde{\otimes}_{\varepsilon} Z\right)^{\odot}$.

To prove the reverse inclusion, we first claim that $\left(Z \tilde{\otimes}_{\varepsilon} X\right)^{\odot} \subset Z^{*} \tilde{\otimes}_{\pi} X^{*}$. For the rest of the proof we fix one $\lambda \in \varrho\left(A_{0}\right)$. For $S \in\left(Z \tilde{\otimes}_{\varepsilon} X\right)^{*}=\mathcal{L}^{i}\left(Z, X^{*}\right)$ we have by Proposition $3.3 R(\lambda, A)^{*}(S)=S \circ R\left(\lambda, A_{0}\right)$. Since $Z$ is sun-reflexive with respect to $T_{0}(t)$, it follows that $R\left(\lambda, A_{0}\right)$ is weakly compact. From a theorem of Grothendieck (see [DU, Thm VIII.4.12]) it follows that $S \circ R\left(\lambda, A_{0}\right)$ is nuclear. Thus $R(\lambda, A)^{*}(S) \in Z^{*} \tilde{\otimes}_{\pi} X^{*}$ and by Proposition 1.1 the claim is proved.

Thus if we fix $S \in \mathcal{L}^{i}\left(Z, X^{*}\right)$, then for arbitrary $\varepsilon>0$ there exist $z_{i} \in Z^{*}$, $x_{i} \in X^{*}$ such that

$$
\left\|S \circ R\left(\lambda, A_{0}\right)-\sum_{i=1}^{n} z_{i}^{*} \otimes x_{i}^{*}\right\|_{i}<\varepsilon .
$$

It follows that

$$
\begin{aligned}
& \left\|S \circ R\left(\lambda, A_{0}\right)^{2}-\sum_{i=1}^{n} R\left(\lambda, A_{0}\right)^{*} z_{i}^{*} \otimes x_{i}^{*}\right\|_{i} \\
& \quad=\left\|\left(S \circ R\left(\lambda, A_{0}\right)-\sum_{i=1}^{n} z_{i}^{*} \otimes x_{i}^{*}\right) \circ R\left(\lambda, A_{0}\right)\right\|_{i}<\varepsilon \cdot\left\|R\left(\lambda, A_{0}\right)\right\| .
\end{aligned}
$$


Since $R\left(\lambda, A_{0}\right)^{*} z_{i}^{*} \in Z^{\odot}$ it follows that $R(\lambda, A)^{* 2}(S)=S \circ R\left(\lambda, A_{0}\right)^{2}$ is in the closed linear subspace of $Z^{*} \tilde{\otimes}_{\pi} X^{*}$ generated by $\left\{z^{*} \otimes x^{*}: z^{*} \in Z^{\odot}, x^{*} \in X^{*}\right\}$. The conclusion now follows from Proposition 1.1.

We point out that the $\pi$-tensor product is not injective, i.e. given a subspace $Y$ of $Z^{*}$, then in general $Y \tilde{\otimes}_{\pi} X^{*}$ cannot be identified with the closed linear subspace of $Z^{*} \tilde{\otimes}_{\pi} X^{*}$ generated by $\left\{y \otimes x^{*}: y \in Y, x^{*} \in X^{*}\right\}$. There are special cases where this is true, e.g. if $Y$ is complemented in $Z^{*}$ or if $X$ is a $C_{0}(\Omega)$-space respectively. Thus we have the following corollary.

CoRollary 3.6: If in addition $Z^{\odot}$ is complemented in $Z^{*}$ or $X=C_{0}(\Omega), \Omega$ locally compact, then $\left(Z \tilde{\otimes}_{\varepsilon} X\right)^{\odot}=Z^{\odot} \tilde{\otimes}_{\pi} X^{*}$.

If $T_{0}(t)$ is a positive semigroup on a Banach lattice $Z$ whose dual has order continuous norm, then by a result of de Pagter (to be published), $Z^{\odot}$ is a projection band in $Z^{*}$. This applies in particular to the case $Z=C_{0}(\Omega)$ and we obtain:

Corollary 3.7: Suppose $T_{0}(t)$ is a positive semigroup on $C_{0}(\Omega)$. Then there exists a measure space $(\tilde{\Omega}, \tilde{\Sigma}, \tilde{\mu})$ such that $C_{0}(\Omega ; X)^{\odot}=L^{1}\left(\tilde{\mu} ; X^{*}\right)$.

Now we consider the case of the $\pi$-tensor product. We are looking for conditions, ensuring that the sun-dual of $X \tilde{\otimes}_{\pi} Z$ can be identified with $Z^{\odot} \tilde{\otimes}_{\varepsilon} X^{*}$. In contrast to Theorem 3.5 now sun-reflexivity (weak compactness of the resolvent) is not sufficient as Example 3.10 below shows. If we require compactness of the resolvent however, then the sun-dual can be described in a nice way.

THEOREM 3.8: Assume that the generator of the semigroup $T_{0}(t)$ on $Z$ has compact resolvent, then for the semigroup induced on $Z \tilde{\otimes}_{\pi} X$ we have $\left(Z \tilde{\otimes}_{\pi} X\right)^{\odot}=$ $Z^{\odot} \tilde{\otimes}_{\varepsilon} X^{*}$.

Proof: As in the proof of Theorem 3.5 it can be shown that $Z \odot \tilde{\otimes}_{\varepsilon} X^{*}$ is contained in the sun-dual of $Z \tilde{\otimes}_{\pi} X$. To prove the converse inclusion we observe that $R\left(\lambda, A_{0}\right)$ being compact implies that for $\varepsilon>0$ there exist $z_{i} \in Z$ and $z_{i}^{*} \in Z^{*}$ such that

$$
\left\|R\left(\lambda, A_{0}\right)-\sum_{i=1}^{m} z_{i}^{*} \otimes z_{i}\right\|<\varepsilon .
$$


Thus given $S \in \mathcal{L}\left(Z, X^{*}\right)$ then

$$
\begin{aligned}
\| S & \circ R\left(\lambda, A_{0}\right)^{2}-\sum_{i=1}^{m} R\left(\lambda, A_{0}\right)^{*} z_{i}^{*} \otimes S z_{i} \| \\
& =\left\|S \circ\left(R\left(\lambda, A_{0}\right)-\sum_{i=1}^{m} z_{i}^{*} \otimes z_{i}\right) \circ R\left(\lambda, A_{0}\right)\right\| \\
& \leq \varepsilon\|S\|\left\|R\left(\lambda, A_{0}\right)\right\| .
\end{aligned}
$$

It follows that $R(\lambda, A)^{* 2}(S)$ can be approximated with respect to the operator norm by elements of $Z^{\odot} \otimes X^{*}$. Since the operator norm induces the $\varepsilon$-norm it follows that $R(\lambda, A)^{* 2}(S) \in Z^{\odot} \tilde{\otimes}_{\varepsilon} X^{*}$ for every $S \in \mathcal{L}\left(Z, X^{*}\right)$. Then from Proposition 1.1 we can conclude that $\left(Z \tilde{\otimes}_{\pi} X\right)^{\odot} \subset Z^{\odot} \tilde{\otimes}_{\varepsilon} X^{*}$.

The case $Z=L^{1}(\mu)$ was already proved in [Pa1]. On spaces $C_{0}(\Omega), \Omega$ locally compact, or spaces $L^{1}(\mu)$, a resolvent is weakly compact if and only it is compact (see $[\mathrm{Pa} 2])$. Therefore the following corollary is an immediate consequence of Theorem 3.8 .

Corollary 3.9: Assume that $Z$ is either a space $L^{1}(\mu)$ or a space $C_{0}(\Omega), \Omega$ locally compact. If the semigroup $T_{0}(t)$ is sun-reflexive then

$$
\left(Z \tilde{\otimes}_{\pi} X\right)^{\odot}=Z^{\odot} \tilde{\otimes}_{\varepsilon} X^{*} .
$$

In general weak compactness of the resolvent is not enough in Theorem 3.8, as the following example shows.

Example 3.10: Consider the semigroup of translations on $Z=L^{P}(\mathbb{R})$. For $1<p<\infty$ we have $L^{p}(\mathbb{R})^{\odot}=L^{p}(\mathbb{R})^{*}=L^{q}(\mathbb{R})$ with $1 / p+1 / q=1$ and the resolvent is weakly compact, $Z$ being reflexive. Assuming that

$$
\left(L^{p}(\mathbb{R}) \tilde{\otimes}_{\pi} X\right)^{\odot}=L^{q}(\mathbb{R}) \tilde{\otimes}_{\varepsilon} X^{*}=\left\{T \in \mathcal{L}\left(L^{p}(\mathbb{R}), X^{*}\right): T \text { is compact }\right\}
$$

then from Proposition 3.3 and Proposition 1.1 we conclude that $S \circ R\left(\lambda, A_{0}\right)$ is compact for every $S \in \mathcal{L}\left(L^{p}(\mathbb{R}), X^{*}\right)$. Choosing $X=L^{q}(\mathbb{R})$ and $S$ the identity on $L^{p}(\mathbb{R})$ shows that $R\left(\lambda, A_{0}\right)$ has to be compact, which is not the case (for then $A_{0}$ must have countable spectrum, but it is well-known that $\left.\sigma\left(A_{0}\right)=i \mathbb{R}\right)$.

In case $p=1$ the resolvent of the translation group even fails to be weakly compact and the conclusion of Theorem 3.8 again does not hold, as we will now show. 
THEOREM 3.11: If $T_{0}(t)$ is the translation group on $L^{1}(\mathbb{R})$ then $L^{1}(\mathbb{R} ; X)^{\odot}=$ $B U C\left(\mathbb{R} ; X^{*}\right)$.

Proof: First we claim that $R\left(\lambda, A_{0}\right)$ is representable [Pa1]. For almost all $s$ we have

$$
\begin{aligned}
\left(R\left(\lambda, A_{0}\right) f\right)(s) & =\int_{0}^{\infty} e^{-\lambda t} f(s+t) d t \\
& =\int_{-\infty}^{\infty} e^{-\lambda(t-s)} \chi_{[s, \infty)}(t) f(t) d t
\end{aligned}
$$

Define $g: \mathbb{R} \rightarrow L^{1}(\mathbb{R})$ by $(g(t))(s)=e^{-\lambda(t-s)} \chi_{[s, \infty)}(t)$. We have

$$
\|g(t)\|_{L^{1}(\mathbb{R})}=\int_{-\infty}^{\infty} e^{-\lambda(t-s)} \chi_{[s, \infty)}(t) d s=\int_{-\infty}^{t} e^{-\lambda(t-s)} d s=\frac{1}{\lambda}
$$

Since also $g$ is continuous as a map $\mathbb{R} \rightarrow L^{1}(\mathbb{R})$, hence in particular strongly measurable, this shows that $g \in L^{\infty}\left(\mathbb{R} ; L^{1}(\mathbb{R})\right)$ and our claim is proved. From Proposition 2.2 in $[\mathrm{Pa} 1]$ we deduce that $L^{1}(\mathbb{R} ; X)^{\odot} \subset L^{\infty}\left(\mathbb{R} ; X^{*}\right)$. Let $h \in$ $L^{1}(\mathbb{R} ; X)^{\odot}$. We claim that $h$ is continuous. Let $\phi_{n}$ be any continuous function with compact support such that $\phi_{n}(t)=1$ for all $t \in[-n, n]$. Clearly it suffices to prove that $h \phi_{n}$ is continuous for all $n$. Since each $h \phi_{n}$ is compactly supported and since obviously $h \in L^{1}(\mathbb{R} ; X)^{\odot}$ implies $h \phi_{n} \in L^{1}(\mathbb{R} ; X)^{\odot}$, we may consider $h \phi_{n}$ as an element of $L^{1}\left(\left[-N_{n}, N_{n}\right] ; X\right)^{\odot}$ for some $N_{n}$ large enough. Since $L^{1}\left(\left[-N_{n}, N_{n}\right]\right)$ is $\odot$-reflexive with respect to translation (see e.g. [HPh]) we have by Theorem 3.9 that

$$
\begin{aligned}
L^{1}\left(\left[-N_{n}, N_{n}\right] ; X\right)^{\odot} & =L^{1}\left(\left[-N_{n}, N_{n}\right]\right)^{\odot} \tilde{\otimes}_{\varepsilon} X^{*} \subset C\left(\left[-N_{n}, N_{n}\right]\right) \tilde{\otimes}_{\varepsilon} X^{*} \\
& =C\left(\left[-N_{n}, N_{n}\right] ; X^{*}\right) .
\end{aligned}
$$

Hence $h \phi_{n} \in C\left(\left[-N_{n}, N_{n}\right] ; X^{*}\right)$. This proves that $L^{1}(\mathbb{R} ; X)^{\odot} \subset C\left(\mathbb{R} ; X^{*}\right)$. But then we must have that actually $h \in B U C\left(\mathbb{R} ; X^{*}\right): h$ is bounded as an element of $L^{\infty}\left(\mathbb{R} ; X^{*}\right)$, and uniformly continuous since otherwise the map $t \mapsto T^{*}(t) h$ is easily seen not to be norm-continuous. This shows $L^{1}(\mathbb{R} ; X)^{\odot} \subset B U C\left(\mathbb{R} ; X^{*}\right)$. The reverse inclusion holds trivially.

This theorem is the $L^{1}$-analogue of Theorem 2.2. Now in general it is not true that

$$
B U C(\mathbb{R} ; X)=B U C(\mathbb{R}) \tilde{\otimes}_{\varepsilon} X
$$


holds. In fact, any function in $B U C(\mathbb{R}) \tilde{\otimes}_{\varepsilon} X$ must have relatively compact range whereas it is easy to construct functions in $B U C\left(\mathbb{R} ; C_{0}(\mathbb{R})\right)$ not having relatively compact range. Just let $f \in C_{0}(\mathbb{R})$ be any non-zero function. Then the set of translates $\{T(t) f: t \in \mathbb{R}\}$ is not relatively compact, so by defining $F(t)=T(t) f$ we obtain an $F \in B U C\left(\mathbb{R} ; C_{0}(\mathbb{R})\right)$ which does not have relatively compact range.

Remark 3.12: (a) The above examples show that for translation on $Z=L^{p}(\mathbb{R})$, $1 \leq p<\infty$ the conclusion of Theorem 3.8 does not hold for every $X$.

In fact, let $Z$ be any fixed Banach space and let $T_{0}(t)$ be a $C_{0}$-semigroup on $Z$ with generator $A_{0}$. We claim that if for every $X$ the formula $\left(Z \tilde{\otimes}_{\pi} X\right)^{\odot}=$ $Z^{\odot} \tilde{\otimes}_{\varepsilon} X^{*}$ holds, then $R\left(\lambda, A_{0}\right)$ must be compact. Take $X=Z^{*}$. Let $X=Z^{*}$ and assume $\left(Z \tilde{\otimes}_{\pi} X\right)^{\odot}=Z^{\odot} \tilde{\otimes}_{\varepsilon} X^{*}$. Then $R(\lambda, A)^{*}(T)=T \circ R\left(\lambda, A_{0}\right)$ is a compact operator for every $T \in\left(Z \tilde{\otimes}_{\pi} X\right)^{*}=\mathcal{L}\left(Z, X^{*}\right)=\mathcal{L}\left(Z, Z^{* *}\right)$. In particular, letting $T: Z \rightarrow Z^{* *}$ be the canonical embedding, it follows that $R\left(\lambda, A_{0}\right)$ itself is compact. See also [Pa1], where $X=l^{\infty}$ is taken.

(b) Concerning 3.5 the situation is different and weak compactness of $R\left(\lambda, A_{0}\right)$ is not necessary in order that $\left(Z \tilde{\otimes}_{\varepsilon} X\right)^{\odot}=\overline{Z \odot \otimes X^{*}} Z^{*} \bar{\otimes}_{\pi} X^{*}$ holds for every Banach space $X$. In fact, an inspection of the proof of Theorem 3.5 shows that a necessary and sufficient condition for this is that $T \circ R\left(\lambda, A_{0}\right)$ is nuclear for every operator $T \in \mathcal{L}^{i}\left(Z, X^{*}\right)$. An example of a semigroup without weakly compact resolvent but satisfying this condition (by Theorem 2.2 !) is translation in $C_{0}(\mathbb{R})$.

By combining 3.5 and 3.8 one can under suitable assumptions describe the bi-sun-dual of the $\varepsilon$ - and the $\pi$-tensor product. In order to apply 3.5 and 3.8 we formally need the assumption that $Z^{\odot *}$ has the a.p. The proof below however shows that it suffices to have that $Z^{*}$ has the a.p.

For $L^{1}(\mu) \tilde{\otimes}_{\pi} X$ the following result was first proved by de Pagter (unpublished).

Proposition 3.13: Suppose $R\left(\lambda, A_{0}\right)$ is compact. Then:

(i) $\left(Z \tilde{\otimes}_{\pi} X\right)^{\odot \odot}$ is the closure in $Z^{\odot *} \tilde{\otimes}_{\pi} X^{* *}$ of $Z \otimes X^{* *}$. If either $Z$ is complemented in $Z^{\odot *}$ or $X$ is an $L^{1}(\mu)$-space then $\left(Z \tilde{\otimes}_{\pi} X\right)^{\odot \odot}=Z \tilde{\otimes}_{\pi} X^{* *}$.

(ii) If either $Z^{\odot}$ is complemented in $Z^{*}$ or $X=C_{0}(\Omega)$, $\Omega$ locally compact Hausdorff, then $\left(Z \tilde{\otimes}_{\varepsilon} X\right)^{\odot \odot}=Z \tilde{\otimes}_{\varepsilon} X^{* *}$.

Proof: First we prove (ii). By Corollary 3.6 we have $\left(Z \tilde{\otimes}_{\varepsilon} X\right)^{\odot}=Z^{\odot} \tilde{\otimes}_{\pi} X^{*}$. The conclusion now follows from Theorem 3.8 in case $Z^{\odot *}$ has the a.p. However, inspection of the proof of Theorem 3.8 shows that the a.p. was needed for showing that $R\left(\lambda, A_{0}\right)$ could be approximated by finite rank operators in the 
uniform operator topology. Hence what we must show in the present case is that $R\left(\lambda, A_{0}^{\odot}\right)$ can be approximated by finite rank operators. That this is true when $Z^{*}$ has the a.p., i.e. under Assumption 3.4 (regardless whether $Z^{\odot *}$ has the a.p.), is shown by the following argument. Fix $\lambda \in \varrho\left(A_{0}\right)$. Since $Z^{*}$ has the a.p., $R\left(\lambda, A_{0}\right)$ is the uniform limit of finite rank operators $\Phi_{n} \in Z^{*} \otimes Z$. Then for $\mu \in \varrho\left(A_{0}\right)$, $R\left(\lambda, A_{0}\right) R\left(\mu, A_{0}\right)$ is the uniform limit of $\Phi_{n} R\left(\mu, A_{0}\right)$. Since $R\left(\mu, A_{0}\right)^{*} Z^{*} \subset Z^{\odot}$ it follows that $\Phi_{n} R\left(\mu, A_{0}\right) \in Z^{\odot} \otimes Z$. Moreover,

$$
\left\|R\left(\lambda, A_{0}\right)^{*} R\left(\mu, A_{0}\right)^{*}-\left(\Phi_{n} R\left(\mu, A_{0}\right)\right)^{*}\right\|=\left\|R\left(\mu, A_{0}\right) R\left(\lambda, A_{0}\right)-\Phi_{n} R\left(\mu, A_{0}\right)\right\|,
$$

hence $\mu R\left(\lambda, A_{0}^{\odot}\right) R\left(\mu, A_{0}^{\odot}\right)=\left.\mu R\left(\lambda, A_{0}\right)^{*} R\left(\mu, A_{0}\right)^{*}\right|_{Z \odot}$ is the uniform limit of $\left.\mu \Phi_{n} R\left(\mu, A_{0}\right)^{*}\right|_{Z \odot} \in Z \otimes Z^{\odot} \subset Z^{\odot *} \otimes Z^{\odot}$. Since

$$
R\left(\lambda, A_{0}^{\odot}\right)=\lim _{\mu \rightarrow \infty} \mu R\left(\lambda, A_{0}^{\odot}\right) R\left(\mu, A_{0}^{\odot}\right)
$$

in the uniform operator topology (this follows from the resolvent equation for $A_{0}^{\odot}$ ), we can conclude that $R\left(\lambda, A_{0}^{\odot}\right)$ can be approximated by finite rank operators. As we noted above, from these considerations we can conclude that

$$
\left(Z^{\odot} \tilde{\otimes}_{\pi} X^{*}\right)^{\odot}=Z^{\odot \odot \tilde{\otimes}_{\varepsilon} X^{* *}},
$$

and since $R\left(\lambda, A_{0}\right)$ is compact we have $Z \odot \odot=Z$, and (ii) is proved.

The first assertion of (i) is proved by a similar argument. Now suppose that $Z$ is complemented in $Z^{\odot *}$. Then trivially every $T \in \mathcal{L}\left(Z, X^{*}\right)$ admits an extension to an operator in $\mathcal{L}\left(Z^{\odot *}, X^{*}\right)$. Also, if $X$ is an $L^{1}(\mu)$-space, then $X^{*}$ is injective [LT] and this again implies that every $T \in \mathcal{L}\left(Z, X^{*}\right)$ admits an extension to an operator in $\mathcal{L}\left(Z^{\odot *}, X^{*}\right)$. In other words, in either case the natural map (induced by restriction $\pi: Z^{\odot *} \rightarrow Z$ )

$$
\pi: \mathcal{L}\left(Z^{\odot *}, X^{*}\right) \rightarrow \mathcal{L}\left(Z, X^{*}\right)
$$

is surjective. But since $\mathcal{L}\left(Y, X^{*}\right)=\left(Y \tilde{\otimes}_{\pi} X\right)^{*}$ this shows that the canonical inclusion map

$$
j: Z \tilde{\otimes}_{\pi} X \rightarrow Z^{\odot *} \tilde{\otimes}_{\pi} X
$$

is an embedding. Applying this to $X^{* *}$ instead of $X$ (and noting that $X^{* * *}$ is an $L^{1}(\mu)$-space if $X^{*}$ is) we obtain that $Z \tilde{\otimes}_{\pi} X^{* *}$ can be regarded as a closed subspace of $Z^{\odot *} \tilde{\otimes}_{\pi} X^{* *}$ and this proves the second assertion. 


\section{The $\ell$-Tensor Product}

It is not possible to identify the space $L^{p}(\mu ; X), 1<p<\infty$, with either a $\varepsilon$ - or a $\pi$-tensor product. In this case the so-called $l$-tensor product solves the problem. It was introduced about 1970 by Chaney, Fremlin, Levin and Schaefer [Ch, Fr1, S3]. In order to define it, first of all one has to introduce the class of cone absolutely summing operators. The following result is taken from [S2, IV.3].

Proposition 4.1: Let $Z$ be a Banach lattice, $X$ a Banach space. For a bounded linear map $T: Z \rightarrow X$ the following are equivalent:

(i) $\exists C>0$ such that for every $0 \leq f_{1}, \ldots, f_{n} \in Z, \sum_{i=1}^{n}\left\|T f_{i}\right\| \leq C\left\|\sum_{i=1}^{n} f_{i}\right\|$;

(ii) For every positive sequence $\left(f_{i}\right)$ in $Z$ such that $\sum_{i=1}^{\infty} f_{i}$ converges, the sum $\sum_{i=1}^{\infty}\left\|T f_{i}\right\|$ converges;

(iii) There is an $L^{1}(\mu)$-space such that $T$ admits a factorization $Z \stackrel{T_{1}}{\rightarrow} L^{1}(\mu) \stackrel{T_{2}}{\rightarrow} X$ with $T_{1} \geq 0$;

(iv) $\exists 0 \leq \phi \in Z^{*}$ such that for all $f \in Z,\|T f\| \leq\langle\phi,|f|\rangle$;

(v) The set $\left\{T^{*} x^{*}:\left\|x^{*}\right\| \leq 1\right\}$ is order bounded in $Z^{*}$.

Definition 4.2: $\quad T: Z \rightarrow X$ is called cone absolutely summing (c.a.s.) if one of the equivalent assertions of Proposition 4.1 is satisfied. The set of all c.a.s operators is denoted by $\mathcal{L}^{l}(Z, X)$. For $T \in \mathcal{L}^{l}(Z, X)$ define

$$
\|T\|_{l}:=\inf \{C: \text { (i) in Proposition } 4.1 \text { holds with constant } C\} .
$$

$\mathcal{L}^{l}(Z, X)$ is a Banach space and contains the finite-rank operators. If $X$ is a Banach lattice then $\mathcal{L}^{l}(Z, X)$ is a Banach lattice as well.

The l-nuclear operators $\mathcal{N}^{l}(Z, X)$ are defined as the closure of the finite rank operators in $\mathcal{L}^{l}(Z, X)$.

As a subspace of $\mathcal{L}(Z, X), \mathcal{L}^{l}(Z, X)$ has the following ideal property: given $T \in \mathcal{L}^{l}(Z, X), R \in \mathcal{L}(X)$ and $S \in \mathcal{L}(Z)$ such that its modulus $|S|$ exists, then $R \circ T \circ S \in \mathcal{L}^{l}(Z, X)$ and

$$
\|R \circ T \circ S\|_{l} \leq\|R\|\|T\|_{l}\||S|\| .
$$

Let $u=\sum_{i=1}^{n} z_{i} \otimes x_{i}$. By the formula $T_{u} z^{*}:=\sum_{i=1}^{n}\left\langle z^{*}, z_{i}\right\rangle x_{i}$ we regard $Z \otimes X$ as a linear subspace of $\mathcal{L}^{l}\left(Z^{*}, X\right)$. On $Z \otimes X$ we define the l-norm $\|\cdot\|_{l}$ to be the norm induced by $\mathcal{L}^{l}\left(Z^{*}, X\right)$. The Banach space $Z \tilde{\otimes}_{l} X$ is defined to be the completion of $Z \otimes X$ with respect to the $l$-norm. In this way $Z \tilde{\otimes}_{l} X$ can be identified with the closure of $Z \otimes X$ in the space $\mathcal{L}^{l}\left(Z^{*}, X\right)$. 
In this way $Z^{*} \tilde{\otimes}_{l} X$ can be identified with the closure of $Z^{*} \otimes X$ in $\mathcal{L}^{l}\left(Z^{* *}, X\right)$. Now elements $u=\sum_{i=1}^{n} z_{i}^{*} \otimes x_{i} \in Z^{*} \otimes X$ can also be identified with an operator $\tilde{T}_{u}: Z \rightarrow X$ (rather than $Z^{* *} \rightarrow X$ ), by

$$
\tilde{T}_{u}(z)=\sum_{i=1}^{n}\left\langle z_{i}^{*}, z\right\rangle x_{i} .
$$

The following proposition states that indeed $Z^{*} \tilde{\otimes}_{l} X$ becomes in this way the closure of $Z^{*} \otimes X$ in $\mathcal{L}^{l}(Z, X)$. In fact, the $\mathcal{L}^{l}(Z, X)$-closure of $Z^{*} \otimes X$ is precisely $\mathcal{N}^{\prime}(Z, X)$.

Proposition 4.3: $Z^{*} \tilde{\otimes}_{l} X$ can be identified isometrically with $\mathcal{N}^{l}(Z, X)$.

Proof: By definition, $\mathcal{N}^{l}(Z, X)$ is the closure of the finite rank operators in $\mathcal{L}^{l}(Z, X)$. Regarding a finite rank operator $Z \rightarrow X$ as an element of $Z^{*} \otimes X$ as above, we see that $\mathcal{N}^{l}(Z, X)$ is the closure of $Z^{*} \otimes X$ in $\mathcal{L}^{l}(Z, X)$. On the other hand, by definition $Z^{*} \tilde{\otimes}_{1} X$ is the $\mathcal{L}^{l}\left(Z^{* *}, X\right)$-closure of $Z^{*} \otimes X$. Therefore it suffices to show that the $\mathcal{L}^{l}(Z, X)$-norm and the $\mathcal{L}^{l}\left(Z^{* *}, X\right)$-norm agree on $Z^{*} \otimes X$. To this end, let $u \in Z^{*} \otimes X$ be given. On the one hand, we can consider $u$ as a c.a.s. $\operatorname{map} T_{u}: Z^{* *} \rightarrow X$. This map is also c.a.s. as a map $Z^{* *} \rightarrow X^{* *}$ and

$$
\left\|T_{u}\right\|_{\mathcal{L}^{\prime}\left(Z * Z^{*} X\right)}=\left\|T_{u}\right\|_{\mathcal{L}^{\prime}(Z * *, X * *)} .
$$

On the other hand we may regard $u$ as a c.a.s. map $\tilde{T}_{u}: Z \rightarrow X$. In this case $\tilde{T}_{u}^{* *}: Z^{* *} \rightarrow X^{* *}$ is c.a.s. [S2, IV Cor. 3.8] and

$$
\left\|\tilde{T}_{u}\right\|_{\mathcal{L}^{\prime}(Z, X)}=\left\|\tilde{T}_{u}^{* *}\right\|_{\mathcal{L}^{\prime}\left(Z^{* *}, X * *\right)}
$$

But clearly as maps $Z^{* *} \rightarrow X^{* *}$ we have $T_{u}=\tilde{T}_{u}^{* *}$, so combining the two above equalities gives the desired result.

The map $j: L^{p}(\mu) \otimes X \rightarrow L^{p}(\mu ; X), 1 \leq p<\infty$, defined by $j(f \otimes x)(t)=$ $f(t) x$ extends to an isometric isomorphism from $L^{p}(\mu) \tilde{\otimes}_{l} X$ onto $L^{p}(\mu ; X)$. In a similar way one has $C_{0}(\Omega) \tilde{\otimes}_{l} X=C_{0}(\Omega ; X)$. This is summarized in the following proposition [S2, IV.7 Examples 1,4].

Proposition 4.4: One has $L^{p}(\mu ; X)=L^{p}(\mu) \tilde{\otimes}_{1} X, 1 \leq p<\infty$, and $C_{0}(\Omega ; X)=$ $C_{0}(\Omega) \tilde{\otimes}_{1} X$.

One of the surprising properties of the $l$-tensor product is that the dual is given by the same class of operators which is used to define it (the l-norm is 'self-dual'). 
More precisely, one has [S2, IV.7.4]

$$
\left(Z \tilde{\otimes}_{l} X\right)^{*}=\mathcal{L}^{l}\left(Z, X^{*}\right)
$$

Now we want to describe the sun-dual of $Z \tilde{\otimes}_{l} X$ with respect to semigroups induced by a semigroup on one of the factors. Since (in contrast to the $\varepsilon$ - and $\pi$ tensor product) the $l$-tensor product is not symmetric (even when $X$ is a Banach lattice as well) we have to distinguish the two cases where $T_{0}(t)$ is given on $Z$ or on $X$.

First we consider the case where we are given a $C_{0}$-semigroup $T_{0}(t)$ on $X$ with generator $A_{0}$. As in Section 3,id $\otimes T_{0}(t):=\mathrm{id} Z \otimes T_{0}(t)$ extends to a $C_{0}$-semigroup on $Z \tilde{\otimes}_{l} X$.

THEOREM 4.5: Each of the following conditions implies $\left(Z \tilde{\otimes}_{l} X\right)^{\odot}=Z^{*} \tilde{\otimes}_{l} X^{\odot}$ :

(i) $R\left(\lambda, A_{0}\right)$ is compact;

(ii) $R\left(\lambda, A_{0}\right)$ is weakly compact and $Z$ does not contain a sublattice isomorphic to $\ell^{1}$.

Proof: The inclusion $\supset$ can be proved as in 3.5.

For $T \in \mathcal{L}^{l}\left(Z, X^{*}\right)$ one has as in Proposition 3.3 that

$$
R(\lambda, A)^{*}(T)=R\left(\lambda, A_{0}\right)^{*} \circ T
$$

Hence to prove the converse inclusion by Proposition 4.3 we have to show that $R\left(\lambda, A_{0}\right)^{*} \circ T$ is $l$-nuclear as a mapping $Z \rightarrow X^{\odot}$.

(i) Since $T: Z \rightarrow X^{*}$ is c.a.s, by Proposition 4.1(iii) $T$ has a factorization

$$
Z \stackrel{T_{1}}{\rightarrow} L^{1}(\mu) \stackrel{T_{2}}{\rightarrow} X
$$

with $T_{1} \geq 0$. Hence $R\left(\lambda ; A_{0}\right)^{*} \circ T$ factorizes as

$$
Z \stackrel{T_{1}}{\rightarrow} L^{1}(\mu) \stackrel{T_{2}^{\prime}}{\rightarrow} X
$$

with $T_{2}^{\prime}=R\left(\lambda, A_{0}\right)^{*} \circ T_{2}$ compact and taking values in $X^{\odot}$. Thus by [S2, Prop. IV.8.2] $R\left(\lambda, A_{0}\right)^{*} \circ T: Z \rightarrow X^{\odot}$ is $l$-nuclear. 
(ii) By a result due to Schlotterbeck-Lotz (personal communication), if $Y$ is reflexive and $Z$ contains no sublattice isomorphic to $\ell^{1}$, then $\mathcal{N}^{l}(Z, Y)=$ $\mathcal{L}^{l}(Z, Y)$. Since by assumption $R\left(\lambda, A_{0}\right)^{*}: X^{*} \rightarrow X^{\odot}$ is weakly compact, by a well-known result of Davis-Figiel-Johnson-Pelczynski [DFJP] there exists a reflexive space $Y$ such that $R\left(\lambda, A_{0}\right)^{*}$ admits the factorization

$$
X^{*} \stackrel{R_{1}}{\longrightarrow} Y \stackrel{R_{2}}{\longrightarrow} X^{\odot}
$$

Since $T$ is c.a.s., the operator $R_{1} \circ T: Z \rightarrow Y$ is c.a.s. as well and we conclude that $R_{1} \circ T$ is $l$-nuclear. Then $R\left(\lambda, A_{0}\right)^{*} \circ T=R_{2} \circ R_{1} \circ T$ is $l$-nuclear as well.

Note that both $Z=C_{0}(\Omega)$ and $Z=L^{p}(\mu), 1<p<\infty$ do not contain $\ell^{1}$ as a sublattice.

Now we will discuss the case where we are given a $C_{0}$-semigroup $T_{0}(t)$ on $Z$. In general for a bounded linear operator $T$ on $Z$, the operator $T \otimes$ id does not admit an extension to a bounded operator on $Z \tilde{\otimes}_{l} X$. If however $T$ possesses a modulus $|T|$, then the extension exists and

$$
\left\|T \tilde{\otimes}_{l} \mathrm{id}\right\| \leq\||T|\|
$$

Therefore in order to be sure that $T_{0}(t) \otimes$ id admits an extension to a $C_{0^{-}}$ semigroup $T(t)=T_{0}(t) \tilde{\otimes}_{l}$ id of bounded operators on $Z \tilde{\otimes}_{l} X$, we will assume that $T_{0}(t)$ is a positive semigroup (see [Na]). Then for $\lambda$ sufficiently large $R\left(\lambda, A_{0}\right)$ is positive, hence $R\left(\lambda, A_{0}\right) \otimes$ id extends to a bounded linear operator on $Z \tilde{\otimes}_{1} X$. One easily shows that this extension equals $R(\lambda, A)$, the resolvent of the generator $A$ of $T(t)$. Similarly as in Proposition 3.3 one has that $R(\lambda, A)^{*}$ considered as an operator on $\mathcal{L}^{l}\left(Z, X^{*}\right)=\left(Z \tilde{\otimes}_{l} X\right)^{*}$ is given by

$$
R(\lambda, A)^{*}(T)=T \circ R\left(\lambda, A_{0}\right) .
$$

In order to be able to identify $\left(Z \tilde{\otimes}_{l} X\right)^{\odot}$ with $Z^{\odot} \tilde{\otimes}_{l} X^{*}$ we need a certain compactness property of $R\left(\lambda, A_{0}\right)$ which we will describe next.

Definition 4.6: An operator $T \in \mathcal{L}(Z)$ is called $r$-compact if its modulus $|T|$ exists and there is a sequence of finite rank operators $\Phi_{n} \in Z^{*} \otimes Z$ such that

$$
\lim _{n \rightarrow \infty}\left\|\left|T-\Phi_{n}\right|\right\|=0
$$


The adjoint of an $r$-compact operator is $r$-compact again. Since $\|T\| \leq\||T|\|$, every $r$-compact operator is compact. In case $Z=L^{1}(\mu)$ or $Z=C_{0}(\Omega)$ the converse is true (see [S2]). For $Z=L^{2}(\mu)$ the situation is different. In [Fr2] an example is given of a positive compact operator on $L^{2}(\mu)$ which is not $r$-compact. However, in $L^{2}(\mu)$ every Hilbert-Schmidt operator is $r$-compact.

Note that a sufficient condition for $r$-compactness for a positive $T$ is the existence of a positive sequence $\Phi_{n}$ of finite rank operators satisfying $0 \leq \Phi_{n} \leq T$ and $\left\|T-\Phi_{n}\right\| \rightarrow 0$. This is a convenient criterion to check, e.g., whether kernel operators are $r$-compact.

TheOREM 4.7: Suppose $T_{0}(t)$ is a positive $C_{0}$-semigroup on a Banach lattice $Z$ whose resolvent $R\left(\lambda, A_{0}\right)$ is $r$-compact for sufficiently large $\lambda$. Then $\left(Z \tilde{\otimes}_{1} X\right)^{\odot}$ is the closure in $Z^{*} \tilde{\otimes}_{l} X^{*}$ of $Z^{\odot} \otimes X^{*}$. If $Z^{\odot}$ is a sublattice of $Z^{*}$ then $\left(Z \tilde{\otimes}_{l} X\right)^{\odot}=$ $Z \odot \tilde{\otimes}_{1} X^{*}$.

Proof: As before, we will show that $R(\lambda, A)^{2 *}\left(\mathcal{L}^{l}\left(Z, X^{*}\right)\right) \subset \overline{\operatorname{span}}\left(Z^{\odot} \otimes X^{*}\right)$, the closure taken in $Z^{*} \tilde{\otimes}_{l} X^{*}$. By assumption there are finite rank operators $\Phi_{n}$ satisfying $\left\|\left|R\left(\lambda, A_{0}\right)-\Phi_{n}\right|\right\| \rightarrow 0$. Given $T \in \mathcal{L}^{l}\left(Z, X^{*}\right)$ it follows that

$$
\begin{aligned}
\left\|R(\lambda, A)^{2 *}(T)-T \circ \Phi_{n} \circ R\left(\lambda, A_{0}\right)\right\|_{l} & =\left\|T \circ\left(R\left(\lambda, A_{0}\right)-\Phi_{n_{2}}\right) \circ R\left(\lambda, A_{0}\right)\right\|_{l} \\
& \leq\|T\|_{l}\left\|\left|R\left(\lambda, A_{0}\right)-\Phi_{n}\right|\right\|\left\|R\left(\lambda, A_{0}\right)\right\| \\
& \rightarrow 0 .
\end{aligned}
$$

Moreover if $\Phi_{n}=\sum_{i=1}^{m} z_{i}^{*} \otimes z_{i}$ then $T \circ \Phi_{n} \circ R\left(\lambda, A_{0}\right)=\sum_{i=1}^{m} R\left(\lambda, A_{0}\right)^{*} z_{i}^{*} \otimes T z_{i} \in$ $Z^{\odot} \otimes X^{*}$ and the first part of the theorem is proved. The additional statement is a consequence of the left-injectivity of the $l$-tensor product in the sense that if $Z_{1}$ is a sublattice of $Z_{2}$, then $Z_{1} \tilde{\otimes}_{1} X$ can be identified with a closed subspace of $Z_{2} \tilde{\otimes}_{l} X$ (see [S2]).

By the result of de Pagter mentioned after 3.6, the second statement of 4.7 applies to the case where $Z^{*}$ has order continuous norm.

Corollary 4.8: Suppose $Z$ is a Banach lattice with $Z^{*}$ having order continuous norm and let $T_{0}(t)$ be a positive semigroup on $Z$. If $R\left(\lambda, A_{0}\right)$ is $r$-compact for sufficiently large $\lambda$, then $\left(Z \tilde{\otimes}_{l} X\right)^{\odot \odot}=Z \tilde{\otimes}_{l} X^{* *}$.

Proof: Since $R\left(\lambda, A_{0}\right)$ is $r$-compact, hence compact, we have $Z \odot \odot=Z$. Now since $Z^{*}$ has order continuous norm, by the result of de Pagter $Z^{\odot}$ is a projection 
band in $Z^{*}$. Hence we can apply Theorem 4.7 to find that $\left(Z \tilde{\otimes}_{l} X\right)^{\odot}=Z^{\odot} \tilde{\otimes}_{l} X^{*}$. Moreover, the canonical embedding $Z \rightarrow Z^{\odot *}$ factorizes as $Z \rightarrow Z^{* *} \rightarrow Z^{\odot *}$ where the second map is the adjoint of the inclusion map $i: Z^{\odot} \rightarrow Z^{*}$. But since $Z^{\odot}$ is a band, $i^{*}$ is a lattice homomorphism. Combining this with the embedding $Z \rightarrow Z^{* *}$ it follows that $Z^{\odot \odot}=Z$ is a sublattice of $Z^{\odot *}$. Hence we can apply 4.7 to the positive semigroup $T_{0}^{\odot}(t)$ on $Z^{\odot}$. Note that this semigroup has $r$-compact resolvent as well. Indeed, $R\left(\lambda, A_{0}\right)^{*}: Z^{*} \rightarrow Z^{*}$ is $r$-compact and $Z^{\odot}$ is complemented in $Z^{*}$ by a positive projection.

Weak compactness is not sufficient for the conclusion of Theorem 4.7 to hold: take any uniformly continuous semigroup on $L^{p}(\mu), 1<p<\infty$ and note that in general $L^{p}(\mu ; X)^{*}=\left(L^{p}(\mu) \tilde{\otimes}_{l} X\right)^{*} \neq L^{q}(\mu) \tilde{\otimes}_{l} X^{*}=L^{q}\left(\mu ; X^{*}\right)$.

Remark 4.9: An inspection of the proof of Theorem 4.7 shows that the assumption of $r$-compactness of the resolvent can be weakened to the following assumption: $T \circ R\left(\lambda, A_{0}\right)$ is l-nuclear for every $T \in \mathcal{L}^{l}\left(Z, X^{*}\right)$. This condition is satisfied when e.g. $Z=L^{p}(\mu)(1<p<\infty)$ and the resolvent $R\left(\lambda, A_{0}\right)$ is represented by a positive measurable kernel $k$, i.e.,

$$
\left(R\left(\lambda, A_{0}\right) f\right)(x)=\int k(x, y) f(y) d \mu(y) \quad \text { for } \mu \text {-a.a. } x
$$

where $k$ satisfies the condition

$$
\sup _{x} \int k(x, y)^{q} d \mu(y)<\infty, \quad \frac{1}{p}+\frac{1}{q}=1 .
$$

This can be seen as follows. If $T \in \mathcal{L}^{l}\left(L^{p}(\mu), X^{*}\right)$ then by 4.1 (iv) there exists a function $\phi \in L^{q}(\mu), \phi \geq 0$ such that $\|T f\| \leq\langle\phi,|f|\rangle$ for all $f \in L^{p}(\mu)$. Thus $T$ has an extension to a bounded operator on $L^{1}(\phi d \mu)$, which we clenote by $T_{1}$. Let $i: L^{p}(\mu) \rightarrow L^{1}(\phi d \mu)$ be the canonical embedding. Then $i \circ R\left(\lambda, A_{0}\right)$ is also represented by $k$. In order to show that $i \circ R\left(\lambda, A_{0}\right)$ is $l$-nuclear we have to verify that $k \in L^{q}(\mu) \tilde{\otimes}_{l} L^{1}(\phi d \mu)=L^{q}\left(\mu ; L^{1}(\phi d \mu)\right)$. By Jensen's inequality,

$$
\begin{aligned}
\int\left|\int k(x, y) \phi(x) d \mu(x)\right|^{q} d \mu(y) & \leq \iint k(x, y)^{q} \phi(x)^{q} d \mu(x) d \mu(y) \\
& =\int\left(\int k(x, y)^{q} d \mu(y)\right) \phi(x)^{q} d \mu(x) \\
& \leq\left(\sup _{x} \int k(x, y)^{q} d \mu(y)\right) \cdot\|\phi\|_{q}^{q} .
\end{aligned}
$$


Thus $k \in L^{q}\left(\mu ; L^{1}(\phi d \mu)\right)$ and hence $i \circ R\left(\lambda, A_{0}\right)$ is $l$-nuclear. Then $T \circ R\left(\lambda, A_{0}\right)=$ $T_{1} \circ i \circ R\left(\lambda, A_{0}\right)$ is $l$-nuclear as well.

This criterion can be used for the translation group on $L^{p}(\mathbb{R})(1<p<\infty)$. In this case $R\left(\lambda, A_{0}\right)$ is given by

$$
\left(R\left(\lambda, A_{0}\right) f\right)(x)=\int_{x}^{\infty} e^{\lambda(x-y)} f(y) d y
$$

so $k(x, y)=e^{\lambda(x-y)} \chi_{(x, \infty)}$. Hence for each $x$,

$$
\int_{\mathbb{R}} k(x, y)^{q} d y=\int_{x}^{\infty} e^{\lambda q(x-y)} d y=\frac{1}{\lambda q} .
$$

Therefore we obtain:

THEOREM 4.10: Let $T_{0}(t)$ be the translation group on $L^{p}(\mathbb{R}), 1<p<\infty$. Then $L^{p}(\mathbb{R} ; X)^{\odot}=L^{q}\left(\mathbb{R} ; X^{*}\right)$.

This example shows that the criterion from Remark 4.9 is weaker that the one of Theorem 4.7: for the translation group on $L^{p}(\mathbb{R})$ the resolvent is not compact and therefore certainly not $r$-compact.

We close with an application of Theorems 4.5 and 4.7 to vector valued $L^{p}(\mu)$ spaces.

THEOREM 4.11: Consider a space $L^{p}(\mu), 1<p<\infty$, and an arbitrary Banach space $X$.

(i) Given a $C_{0}$-semigroup $T_{0}(t)$ on $X$ which is sun-reflexive, then the induced semigroup on $L^{p}(\mu ; X)$ is sun-reflexive as well. Moreover,

$$
L^{p}(\mu, X)^{\odot}=L^{q}\left(\mu ; X^{\odot}\right) .
$$

(ii) Given a positive $C_{0}$-semigroup on $L^{p}(\mu)$ with $r$-compact resolvent, then for the semigroup induced on $L^{p}(\mu ; X)$ we have $L^{p}(\mu ; X)^{\odot}=L^{q}\left(\mu ; X^{*}\right)$ and $L^{p}(\mu ; X)^{\odot \odot}=L^{p}\left(\mu ; X^{* *}\right)$.

Proof: (i) $\ell^{1}$ does not embed into the reflexive space $L^{p}(\mu)$. (ii) Since $L^{p}(\mu)$ is reflexive, $L^{p}(\mu)^{\odot}=L^{q}(\mu)$ is a sublattice of $L^{q}(\mu)$. 


\section{References}

[BB] P. L. Butzer and H. Berens, Semigroups of Operators and Approximation, Springer-Verlag, New York, 1967.

[Ch] J. Chaney, Banach lattices of compact maps, Math. Z. 129 (1972), 1-19.

[DFJP] W. J. Davis, T. Figiel, W. B. Johnson and A. Pelczynski, Factoring weakly compact operators, J. Funct. Anal. 17 (1974), 311-327.

[DS] N. Dunford and J. Schwartz, Linear Operators, Part I. General Theory, Interscience, New York, 1958.

[DU] J. Diestel and J. J. Uhl, Vector Measures, Math. Surveys No. 15, Am. Math. Soc., Providence, R.I., 1977.

[Fr1] D. H. Fremlin, Tensor products of Banach lattices, Math. Ann. 211 (1974), 87-106.

[Fr2] D. H. Fremlin, A positive compact operator, Manuscr. Math. 15 (1975), 323327.

[HPh] E. Hille and R. S. Phillips, Functional Analysis and Semi-groups, Am. Math. Soc. Colloq. Publ., Vol. 31, Am. Math. Soc., rev. ed., Providence, R.I., 1957.

[LT] J. Lindenstrauss and L. Tzafriri, Classical Banach Spaces I, Springer-Verlag, Berlin, 1977.

[Na] R. Nagel (ed.), One-parameter Semigroups of Positive Operators, Lecture Notes in Mathematics 1184, Springer-Verlag, Berlin, 1986.

[Ne] J. M. A. M. van Neerven, Hahn-Banach type theorems for adjoint semigroups, Math. Ann. 287 (1990), 63-71.

[P] A. Pazy, Semigroups of Linear Operators and Applications to Partial Differential Equations, Applied Math. Sciences 44, Springer-Verlag, Berlin, 1983.

[Pa1] B. de Pagter, Semigroups in spaces of Bochner integrable functions and their duals, in Semigroup Theory and Applications, Lecture Notes in Pure and Applied Mathematics, Vol. 166, pp. 331-339, Marcel Dekker Inc., New YorkBasel, 1989.

[Pa2] B. de Pagter, A characterization of sun-reflexivity, Math. Ann. 283 (1989), 511-518.

[Pl] A. Plessner, Eine Kennzeichnung der totalstetigen Funktionen, J. reine ang. Math. 160 (1929), 26-32.

[S1] H. H. Schaefer, Topological Vector Spaces, The Macmillan Company, New York, 1966. 
[S2] H. H. Schaefer, Banach Lattices and Positive Operators, Springer-Verlag, Berlin, 1974.

[S3] H. H. Schaefer, Normed tensor products of Banach lattices, Isr. J. Math. 13 (1972), 400-415. 